\title{
THE EFFECTS OF PEDAL RATES ON PEDAL REACTION FORCES DURING ELLIPTICAL EXERCISE
}

\author{
Hui-Lien Chien, Tsung-Yuan Tsai and Tung-Wu Lu* \\ Institute of Biomedical Engineering \\ National Taiwan University, Taiwan \\ *twlu@ntu.edu.tw
}

Accepted 27 July 2007

\begin{abstract}
Despite the growing popularity in recent years of elliptical exercise (EE), little is known regarding the loadings applied to the body during EE. Since overloading to the body may lead to early fatigue of the muscles and increase the incidence of overuse injuries, such information is necessary for safe use of the elliptical trainer (ET) as a fitness tool. The current study aimed to determine the typical patterns and loading rates of the measured pedal reaction forces (PRF), and to quantify their differences from those during level walking, and the effects of pedaling rate. Fifteen male adults performed level walking and EE while 3D marker data, right PRFs and ground reaction forces (GRF) were measured. The parameters of the ET were set for two different pedal rates: $50 \mathrm{rpm}$ and $70 \mathrm{rpm}$. For each pedal rate, the parameters were set to match the variables measured during level walking, with a mean step length of $55 \%$ leg length and no workload. During early stance the vertical PRF was smaller than the GRF, while the medial and posterior PRF were greater. PRFs also occurred during swing. Loading rates around heelstrike during EE were all smaller than those during walking. The medial, anterior and posterior PRF, as well as the medial and vertial loading rates increased with increasing pedal rates. The basic force patterns of $\mathrm{EE}$ and the effects of pedal rate were established in order to determine the true potential for such instrumentation in locomotion analysis. The results will be helpful for future related studies.
\end{abstract}

Keywords: Elliptical trainer; Fitness; Impact; Joint loading; Speed.

\section{INTRODUCTION}

Elliptical trainers (ET) have been used extensively for aerobic exercise in homes, health clubs and rehabilitation facilities. ${ }^{1}$ Many studies have reported that exercising with an ET can improve and maintain cardiovascular fitness. ${ }^{2,3}$ Although the popularity of elliptical exercises (EE) has increased over the past few years, previous studies on EE have focused mainly on their effects on the cardiopulmonary function. ${ }^{1,2,4}$ Little is known regarding the loadings applied to the body during EE. Since overloading to the body may lead to early fatigue of the muscles and increase the incidence of overuse injuries, such information is necessary for proper and safe use of the ET as a fitness tool.

The forces humans exert on the environment, and thus the reaction forces, are a useful measure in the study of motor control because they represent the final common output of the motor system in its effort to perform many common tasks. ${ }^{5}$ The ground reaction force (GRF) is the most common force exerted on the body by the ground during standing, walking or running. Therefore, the GRF has been one of the most valuable biomechanical variables in gait analysis used to identify the loading conditions, the necessary muscle

\footnotetext{
*Corresponding author: Dr. Tung-Wu Lu, Institute of Biomedical Engineering, National Taiwan University, No. 1, Sec. 1, Jen-Ai Road, Taipei 100, Taiwan, R.O.C.
} 
control and the mechanical energy fluctuations during walking. ${ }^{6}$ These reaction forces are merely the algebraic summation of all mass-acceleration products of all body segments ${ }^{7}$ and can be resolved into three components: vertical, anterior-posterior (a-p), and medial-lateral $(\mathrm{m}-\mathrm{l}) .{ }^{8,9}$ One can also calculate the loading of a joint by considering the GRF and the mass-acceleration products of the segments distal to the joint. The basic patterns of reaction forces during locomotion have been studied extensively for over 70 years. ${ }^{6,10,11}$ GRF parameters, such as loading rate, peak impact, maximum thrust and curve shape, have also been used to discuss the effects of age or pathological conditions during common activities. These GRF characteristics during walking were dependent upon numerous external factors, such as the subject's body mass, walking style, area of the foot-ground contact and, especially, walking speed. Many researchers have reported that the impact forces around heelstrike increase with increased walking speed. ${ }^{11,12}$ The correlation between the impact forces during walking and overuse injuries has prompted numerous experimental studies of GRF during the past 20 years. $^{12,13}$

Repetitive impulsive forces at heelstrike have been reported to produce orthopaedic disorders such as joint degeneration, ${ }^{14}$ even though walking has been recognized to have positive effects on health and has become the preferred mode of exercise for millions of people. ${ }^{15,16}$ Elliptical trainers are assumed to offer the benefits of walking without its negative effects (impulsive forces) by providing a walking-like exercise, while maintaining constant foot contact with the pedal platforms. ${ }^{1,4,17}$ Porcari et al. ${ }^{18}$ measured normal pedal reaction force $(\mathrm{PRF})$ during $\mathrm{EE}$ and reported that the peak normal PRFs were similar to the peak vertical reaction forces during treadmill walking, and intermediate to those observed during cycling and treadmill running. Since the shear components were not measured and the pedals were rotating during $\mathrm{EE}$, the actual vertical, a-p and m-l components of the PRF could not be obtained. Therefore, the measured normal PRF cannot represent the actual vertical force applied to the body. Comparisons of the normal PRF with the vertical GRF during other activities may not be appropriate. The loading rates of these force components, which are regarded to be associated with joint injuries, were not reported. Similar to walking, increasing pedaling rates during EE may increase the loading rate. To the best knowledge of the authors, no study has reported the magnitudes and the loading rates of the three-dimensional (3D) PRF during EE, and how these variables change with increased pedaling rate. The purposes of the current study were thus to measure the PRF during EE; to determine the typical patterns and loading rates of the measured PRF; and to quantify their differences from those during level walking and the effects of pedaling rate on these variables.

\section{MATERIALS AND METHODS}

Fifteen adult male subjects with a mean age of 25.5 years (standard deviation: 2.5 years), mean height of $173.7 \mathrm{~cm}$ (standard deviation: $4.9 \mathrm{~cm}$ ), and a mean body mass of $72.7 \mathrm{~kg}$ (standard deviation: $7.9 \mathrm{~kg}$ ) were recruited in this study. None of the participants had any musculoskeletal, cardiovascular, or neurological disorders affecting the activity. Each subject provided written informed consent following the requirements of the Institutional Human Research Ethics Committee.

A commercially available elliptical trainer (Cycling \& Health Tech Industry R\&D Center, Taiwan) was used in this study (Fig. 1). A force transducer (Bertec Corporation, USA) instrumented under the right pedal of the ET was used to measure the three components of the PRF and the position of its point of application at a frequency of $1080 \mathrm{~Hz}$. Two forceplates (Advanced Mechanical Technology Inc, USA), installed in the center of an eight-meter level walkway, were used to measure the GRF at the same sampling frequency during level walking. For determining the temporal-spatial gait variables, including stride length and cadence, two markers were attached to the bilateral heels. Another four markers were placed on the transducer of the right pedal to track the motion of the transducer. The motion of these markers was measured by a seven-camera motion analysis system (Vicon512, Oxford Metrics Group, UK) at a sampling rate of $120 \mathrm{~Hz}$. All the measurements were performed synchronously.

Each subject first performed six successful walking trials at a self-selected pace on an eight-meter walkway, with a successful trial consisting of the entire foot landing on the force plate. The parameters of the ET were then set for two different pedal rates: $50 \mathrm{rpm}$ and $70 \mathrm{rpm}$. A pedal rate of $50 \mathrm{rpm}$ is similar to the cadence during level walking at a self-selected pace, while $70 \mathrm{rpm}$ simulates faster walking. For each pedal rate, the parameters were set to match the variables measured during level walking, with a mean step length of $55 \%$ leg length and no workload. A metronome was used to assist each subject to perform EE at the prescribed pedal rate. Data collection started when the subject reached the correct pedal rate and maintained 


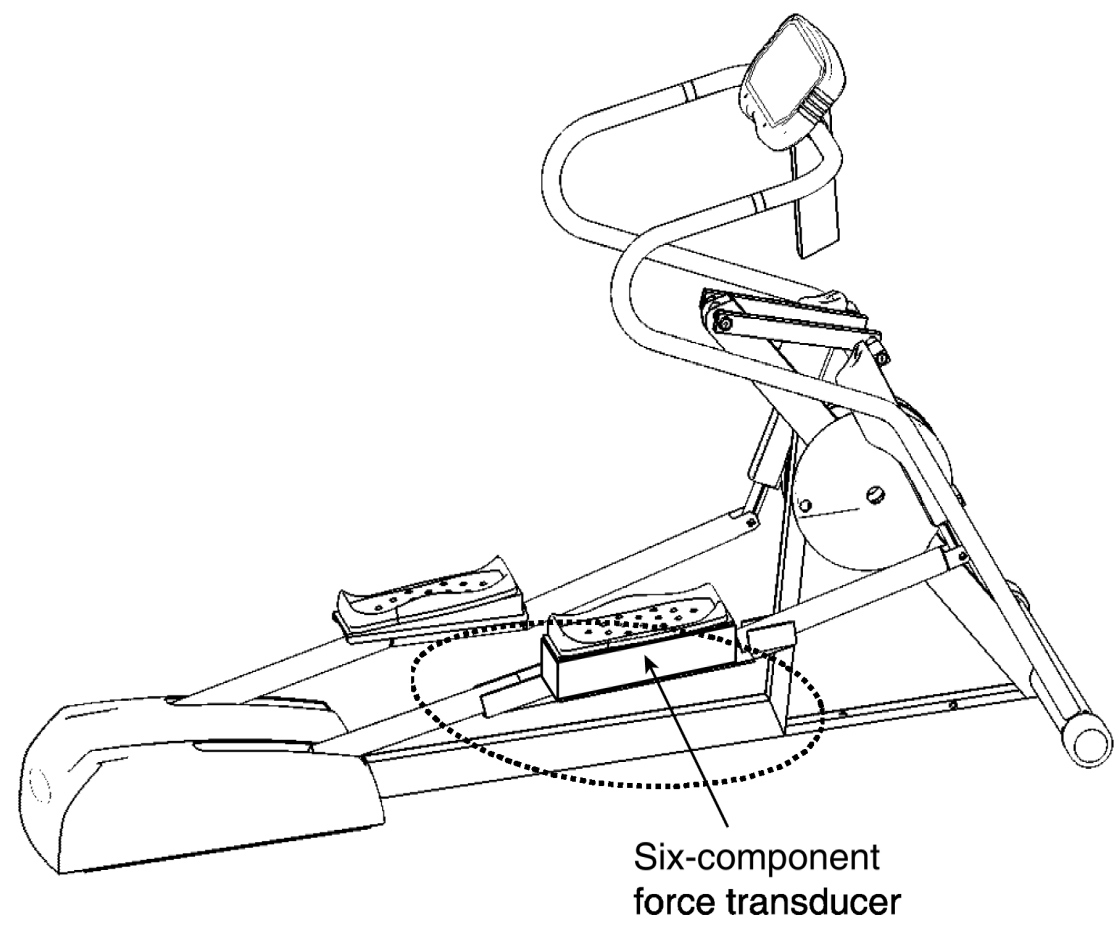

Fig. 1 Experimental set-up showing the elliptical trainer and a six-component force transducer instrumented under the right pedal.

that speed for five seconds. The subjects were allowed to familiarize themselves with the walkway and the elliptical trainer before experimental data were recorded.

In order to remove the inertial effects of the transducer on the measured PRF during motion, the instrumented pedal was unloaded and driven by the non-instrumented pedal to move at a series of pedal rates while the transducer outputs were collected and represented in terms of pedal rate. These values were then subtracted from the measured PRF during tests for subsequent dynamic analysis. In order to determine the magnitude of the peak force and the corresponding time instance precisely, a fourth-order bi-directional Butterworth filter with a cut-off frequency of $10 \mathrm{~Hz}$ was used to low-pass filter the PRF data. The main variables reported in this study are the peak forces and the initial loading for each force component. Initial loading rate was defined as the maximal slope in the PRF and GRF curves between heelstrike and the first peak. The force values and loading rate were then normalized to body weight $(\mathrm{BW})$. The cycle of $\mathrm{EE}$ was divided into two phases based on the pedal positions: stance phase from the most anterior to the most posterior pedal points, and swing phase from the most posterior to the most anterior points.

Comparisons of the calculated variables between the $\mathrm{EE}$ and level walking and the effects of the pedal rate on these variables were tested using a paired $t$-test $(\alpha=0.05)$. SPSS version 10.0 (SPSS Inc., Chicago, IL) was used for all statistical analyses.

\section{RESULTS}

On average, the walking velocity was $112 \mathrm{~cm} / \mathrm{sec}$. Although mean pedal rates and step lengths were slightly lower during EE $(52.20 \pm 2.34 \mathrm{rpm}, 50.56 \pm$ $2.14 \mathrm{~cm})$ than those during level walking $(52.88 \pm$ 9.53 strides $/ \mathrm{min}, 52.17 \pm 3.55 \mathrm{~cm})$, there was no significant difference between these two activities (step length, $\mathrm{p}=0.130$; cadence, $\mathrm{p}=0.616$ ).

The trajectory of the center of pressure (COP) during $\mathrm{EE}$ was different from that during level walking (Fig. 2). The COP moved from heel to toe during walking stance phase, while starting at the midfoot and moving forward close to the head of the metatarsal bones during EE stance phase. At toe-off, the COP shifted toward the medial side during walking, while it shifted toward the lateral side during EE. Consequently, the COP displacements during EE were much smaller than those during walking. In addition, the COP existed during the EE swing phase and moved from forefoot to midfoot.

The patterns of the GRF during walking and PRF during EE were largely different, an apparent difference 


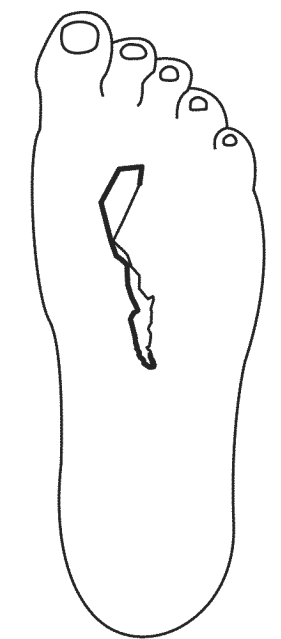

Elliptical Exercise

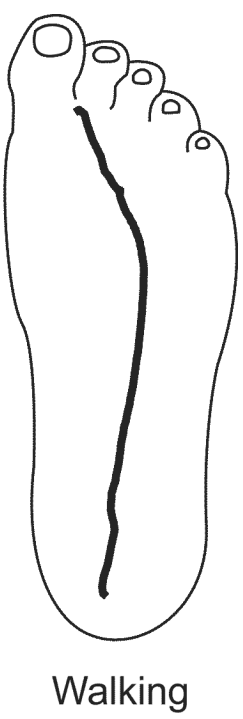

Fig. 2 Center of pressure patterns of a typical subject during $\mathrm{EE}$ and level walking.

being that the GRF was present only during the stance phase, whereas the PRF existed for the entire cycle of EE (Figs. 3 and 4). The vertical and mediolateral components of the GRF during level walking had double peaks, whereas only single peaks were observed in the PRF during EE (Fig. 3). The mediolateral GRF always showed a brief laterally-directed peak at heelstrike, while none was found during EE (Fig. 3). The first peaks of all three component forces occurred before $20 \%$ of the total motion cycle during both level walking and EE.

Compared to walking, the PRF was positioned and directed more posteriorly and medially in the stance phase (Fig. 3). In the early stance the magnitude of the vertical PRF of EE was smaller (EE: 94.44 \pm 9.00\% BW, walking: $103.05 \pm 5.12 \% \mathrm{BW}, \mathrm{p}=0.003)$, while the medial (EE: $8.09 \pm 2.08 \%$ BW, walking: $4.36 \pm$ $2.00 \% \mathrm{BW}, \mathrm{p}<0.0001$ ) and posterior (EE: $15.83 \pm$ $3.13 \%$ BW; walking: $13.61 \pm 2.09 \% \mathrm{BW}, \mathrm{p}=0.03)$ shear forces were greater. As the pedal rate increased from $50 \mathrm{rpm}$ to $70 \mathrm{rpm}$, the peak medial and posterior components in the early stance increased significantly (medial, $\mathrm{p}=0.031$; posterior, $\mathrm{p}=0.0001$ ) (Fig. 4). The peak anterior shear force which occurred in late stance also increased significantly $(\mathrm{p}=0.009)$ (Fig. 4). No significant changes in the magnitude of vertical component were seen as the pedal rate changed (Fig. 4).

During elliptical exercise at $50 \mathrm{rpm}$, the loading rates of the three shear forces around heelstrike were all much smaller than those during walking, Table 1. The loading rates of the medial and vertical components which occurred at heelstrike increased significantly as pedal rates rose from $50 \mathrm{rpm}$ to $70 \mathrm{rpm}$. In addition, at the higher pedal rates the vertical shear force showed a much smaller loading rate compared to that during walking.

\section{DISCUSSION}

The current study is the first investigation to provide a detailed 3D analysis of PRF during EE. Although elliptical trainers are capable of simulating the motion of human gait, obvious differences in force patterns and magnitudes between EE and level walking were demonstrated. A consequence of fundamental differences in movement strategies between the two major forms of human progression (closed and open kinetic chain) may be the reason.

The closed kinetic chain motion with the constrained pedal trajectory during EE seemed to be the main contribution for the different trajectory of the COP compared to level walking. The COP is the result of the inertial forces of the body and restores equilibrium forces in the postural control system. During level walking, the COP started at the heel, while it started at the midfoot during EE. This indicates that initial contact was made by the heel during walking, whereas the average of all the forces was on the midfoot at the beginning of the EE cycle. During the stance phase of walking and EE, the COP progressed forward. However, it reached the toe during walking, while ending close to the head of the metatarsal bones during EE. These differences may affect the performance of joint moments.

The basic force patterns of EE were established in the current study (Fig. 3). All three components of the PRF display sinusoidal patterns with peak vertical, medial and posterior shear forces occurring in the early stance phase. The medial and posterior shear forces occur during the early stance phase, indicating that the PRF is acting backwards and medial as the muscle forces cause the foot to push ahead and towards the outside of the pedal. As the pedal moves to the most posterior point, the pedal is partially unloaded in terms of decreased vertical and medial shear forces. The vertical, medial and posterior shear forces then increase following the beginning of the swing phase. These force patterns were not affected by the pedal rate.

The different patterns of GRF/PRF between level walking and $\mathrm{EE}$ inferred that the segment motion (acceleration) patterns are different during stance in both activities. The reaction forces are the algebraic summation of all mass-acceleration products of all body segments. ${ }^{7}$ Double peaks in the vertical and 

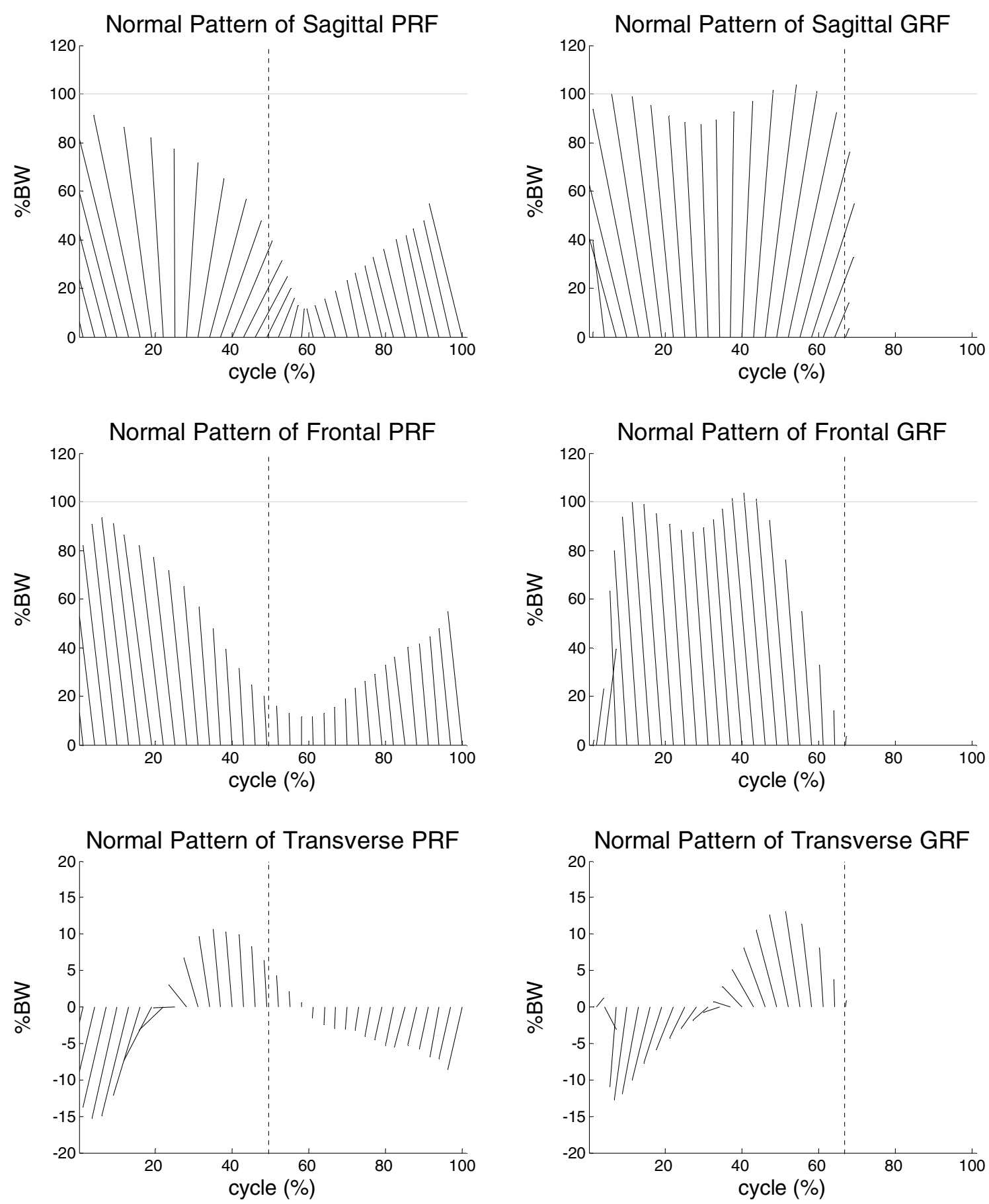

(A)

(B)

Fig. 3 Ensemble-averaged (A) PRF during elliptical exercise and (B) GRF during level walking in the sagittal, frontal and transverse planes. The right directions indicate anterior orientation in the sagittal plane and lateral orientation in the frontal and transverse planes. The vertical directions indicate upward orientation in the sagittal and frontal planes and anterior orientation in the transverse plane.

mediolateral components of the GRF during level walking, whereas only a single peak in the PRF during EE, were observed, implying that lower limb motion in the frontal plane could be different between these two activities. Apart from the segment motion, the weight-sharing from the swing leg may also contribute to these differences. The swing leg assisted in supporting the body weight during elliptical exercise and may thus also reduce the demands on the abductors of the stance limb. 

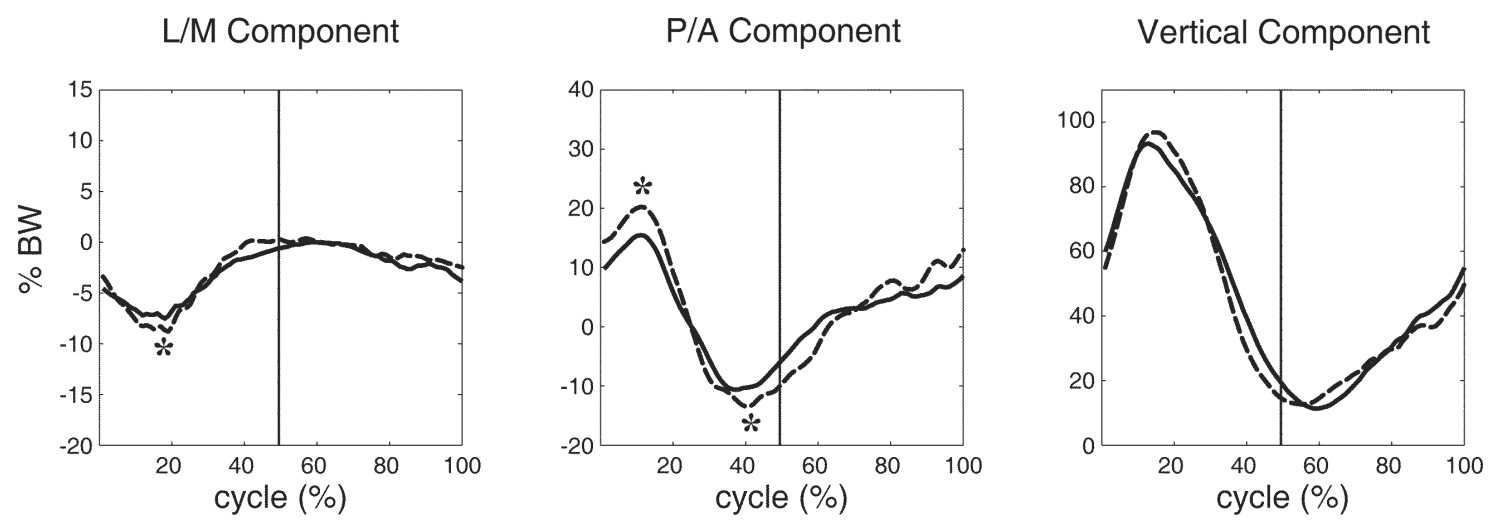

Fig. 4 Ensemble-averaged PRF at $50 \mathrm{rpm}$ (solid lines) and $70 \mathrm{rpm}$ (dashed lines) during elliptical exercise. An asterisk indicates a significant difference between the two pedal rates $(\mathrm{p}<0.05)$. Vertical line indicates the time at which the pedal moves to the posterior point of the pedal trajectory.

Table 1. Means (Standard Deviations) of the GRF During Walking and PRF During EE, Including Vertical, Mediolateral and Anterior-Posterior Components. An Asterisk Indicates a Significant Difference Between the Two Activities or Between Two Pedal Rates $(\mathrm{p}<0.05)$.

\begin{tabular}{|c|c|c|c|c|c|}
\hline \multirow[b]{2}{*}{ Loading Rate $\left(\mathrm{BWs}^{-1}\right)$} & \multirow[b]{2}{*}{ Walking } & \multicolumn{2}{|c|}{ EE } & \multirow{2}{*}{$\begin{array}{c}\text { p value } \\
\text { Walking vs. EE }(50 \mathrm{rpm})\end{array}$} & \multirow{2}{*}{$\begin{array}{c}\mathrm{p} \text { value } \\
50 \mathrm{rpm} \text { vs. } 70 \mathrm{rpm}\end{array}$} \\
\hline & & $50 \mathrm{rpm}$ & $70 \mathrm{rpm}$. & & \\
\hline Vertical & $\begin{array}{c}48.78 \\
(16.98)\end{array}$ & $\begin{array}{c}3.78 \\
(1.75)\end{array}$ & $\begin{array}{c}5.83 \\
(2.79)\end{array}$ & $<0.0001^{*}$ & $0.0006^{*}$ \\
\hline Mediolateral & $\begin{array}{c}4.61 \\
(2.07)\end{array}$ & $\begin{array}{c}0.42 \\
(0.16)\end{array}$ & $\begin{array}{c}0.82 \\
(0.35)\end{array}$ & $<0.0001^{*}$ & $0.0006^{*}$ \\
\hline Anterioposterior & $\begin{array}{c}6.27 \\
(3.03)\end{array}$ & $\begin{array}{c}0.85 \\
(0.50)\end{array}$ & $\begin{array}{c}1.00 \\
(0.89)\end{array}$ & $<0.0001^{*}$ & 0.1996 \\
\hline
\end{tabular}

A smaller maximum vertical PRF and its loading rate at heelstrike during EE, approximately $91.65 \%$ and $7.74 \%$ of level walking, respectively, verified its lower impact loading which may reduce the potential for femoral-tibial joint osteoarthritis. Maintaining both feet in constant contact with the pedal platforms to form a closed kinetic chain during the EE contributed to these advantages. The loading rate values of the vertical component during level walking was much greater than those $\left(7.77 \pm 1.78 \mathrm{BWs}^{-1}\right)$ reported by Keller et al. ${ }^{12}$ because the walking velocity was $1.5 \mathrm{~ms}^{-1}$. Calculation of the loading rate may explain these different results. Keller et al. ${ }^{12}$ calculated loading rate by dividing the first maximum force into the time interval between initial foot contact and the occurrence of the maximum vertical thrust force. However, this will underestimate the loading rate because it ignores the nonlinear portion. Loading rate in the current study was determined by the slope of the force curves, and could provide a better reflection of the loading rate during gait and EE. However, the values reported in the current study are still larger than $30.9 \pm 7.6 \mathrm{BWs}^{-1}$ shown by McCaw et al. ${ }^{19}$ who used the same calculation method.
Barefoot walking in our study, while walking with shoes in the study by McCaw et al.,${ }^{19}$ may be the reason.

The pedal rates affected only the magnitude of the mediolateral and anteroposterior shear forces. It revealed that bigger propulsion and braking forces in the sagittal plane, and bigger inside forces in the frontal plane were needed for bigger pedal rates. Previous study on gait ${ }^{11}$ demonstrated that the three components of the GRF increased with increasing walking speed. It seems that these two activities may adopt different ways to accomplish this task. Different from the magnitude of GRF/PRF, greater pedaling rates increased the loading rate in the vertical and mediolateral components. Although the vertical loading rates increased during $\mathrm{EE}$, the values were still much smaller than those during walking, suggesting that EE provides less stress on the femoral-tibial joint, despite an increase in the pedal rate. Similar results were also found in the other two components. These results explain the predominance in popular preference of EE over level walking.

The motion of EE also resembles standing cycling with a stride more similar to walking. The effects of pedaling speed are well documented in the cycling 
literature. ${ }^{20,21}$ Researchers have reported that changing the cadence during cycling will evoke changes in pedal forces and joint moments. ${ }^{21,22}$ Takaishi et al., ${ }^{23,24}$ suggested that higher pedal rates, coupled with lower pedal reaction forces and fewer fatigable muscle fibers recruited, led to improvements in cycling performance. However, as pedal rates rose from $50 \mathrm{rpm}$ to $70 \mathrm{rpm}$, the mediolateral and anterior-posterior shear forces increased, while the vertical reaction force did not. Weight bearing during EE may contribute to this difference. Hence, the biomechanics during EE may also differ from those during cycling, and the optimal cadences suggested by studies on cycling may not be suitable for EE. Inappropriate pedal rates will increase loading and muscle demand, lead to early fatigue, and may thus decrease fitness training effects. Therefore, continued research is needed on the kinematics and kinetics of $\mathrm{EE}$ to provide complete information about $\mathrm{EE}$ and to establish the guideline for safe usage in the training of healthy users, as well as of patients in the course of rehabilitation.

\section{CONCLUSIONS}

The basic force patterns of EE and the pedal rate effects were established in order to realize the true potential for such instrumentation in locomotion analysis. The results showed that smaller vertical PRFs, while greater medial and posterior shear forces during simulated walking on an elliptical trainer were achieved at smaller loading rates. Increasing pedal rates increased the peaks of the mediolateral and anteroposterior components, suggesting that bigger propulsion, braking and inside forces provided by the stance leg were needed for higher loading rates. Greater loading rates in the mediolateral and vertical components were also found. The results will be helpful for future related studies.

\section{ACKNOWLEDGMENTS}

The authors would like to thank the Cycling \& Health Tech Industry R\&D Center for supporting the work reported in this paper.

\section{REFERENCES}

1. Batte AL, Darling J, Evans J, Lance LM, Olson EI, Pincivero DM, Physiologic response to a prescribed rating of perceived exertion on an elliptical fitness crosstrainer, J Sports Med Phys Fitness 43:300-305, 2003.

2. Egana M, Donne B, Physiological changes following a 12 week gym based stair-climbing, elliptical trainer and treadmill running program in females, $J$ Sports Med Phys Fitness 44:141-146, 2004.
3. Kravitz L, Wax B, Mayo JJ, Daniels R, Charette K, Metabolic response of elliptical exercise training, J Sports Med Phys Fitness 30:S169, 1998.

4. Green JM, Crews TR, Pritchett RC, Mathfield C, Hall L, Heart rate and ratings of perceived exertion during treadmill and elliptical exercise training, Percept Mot Skills 98:340-348, 2004.

5. Rogers LM, Brown DA, Gruben KG, Foot force direction control during leg pushes against fixed and moving pedals in persons post-stroke, Gait Posture 19:58-68, 2004.

6. Gottschall JS, Kram R, Ground reaction forces during downhill and uphill running, J Biomech 38:445-452, 2005.

7. Winter DA, Kinetics, Forces and Moments of Force, in Biomechanics and Motor Control of Human Movement, John Wiley \& Sons, New York, pp. 96-100, 2005.

8. Perry J, Ground reaction force and vector analysis, in Slack (ed.), Gait Analysis: Normal and Pathological Function, Thorofare, pp. 414-429, 1992.

9. Cook TM, Farrell KP, Carey IA, Gibbs JM, Wiger GE, Effects of restricted knee flexion and walking speed on the vertical ground reaction force during gait, J Orthop Sports Phys Ther 25:236-244, 1997.

10. Fenn W, Work against gravity and work due to velocity changes in running, Am J Physiol 93:433-462, 1930.

11. Nilsson J, Thorstensson A, Ground reaction forces at different speeds of human walking and running, Acta Physiol Scand 136:217-227, 1989.

12. Keller TS, Weisberger AM, Ray JL, Hasan SS, Shiavi RG, Spengler DM, Relationship between vertical ground reaction force and speed during walking, slow jogging, and running, Clin Biomech 11:253-259, 1996.

13. Miller DI, Ground reaction forces in distance running, in Cavanagh PR (ed.), Biomechanics of Distance Running, Human Kinetics, Champaign, IL, pp. 203-224, 1990.

14. Collins JJ, Whittle MW, Impulsive forces during walking and their clinical implications, Clin Biomech 4:179-187, 1989.

15. Simons R, Andel R, The effects of resistance training and walking on functional fitness in advanced old age, J Aging Health 18:91-105, 2006.

16. Voloshin A, The influence of walking speed on dynamic loading on the human musculoskeletal system, Med Sci Sports Exerc 32:1156-1159, 2000.

17. Lu TW, Chien HL, Chen HL, Joint loading in the lower extremities during elliptical exercise, Med Sci Sports Exerc, 2007.

18. Porcari JP, Zedaker JM, Nawer I, Miller M, Evaluation of an elliptical exercise in comparison to treadmill walking and running, stationary cycling, and stepping, Med Sci Sports Exerc 30:S168, 1998.

19. McCaw ST, Heil ME, Hamill J, The effect of comments about shoe construction on impact forces during walking, Med Sci Sports Exerc 32:1258-1264, 2000.

20. Baum BS, Li L, Lower extremity muscle activities during cycling are influenced by load and frequency, $J$ Electromyogr Kinesiol 13:181-190, 2003. 
21. Redfield R, Hull ML, On the relation between joint momentum and pedalling rates at constant power in bicycling, J Biomech 19:317-329, 1986.

22. Hull ML, Jorge M, A method for biomechanical analysis of bicycle pedaling, J Biomech 18:631-644, 1985.

23. Takaishi T, Yasuda Y, Ono T, Moritani T, Optimal pedaling rate estimated from neuromuscular fatigue for cyclists, Med Sci Sports Exerc 28:1492-1497, 1996.

24. Takaishi T, Yamamoto T, Ono T, Ito T, Moritani T, Neuromuscular, metabolic, and kinetic adaptations for skilled pedaling performance in cyclists, Med Sci Sports Exerc 30:442-449, 1998. 